\title{
FINITE VOLUME APPROXIMATION OF THE EFFECTIVE DIFFUSION MATRIX: THE CASE OF INDEPENDENT BOND DISORDER ${ }^{\star}$
}

\author{
Pietro CAPUTO $^{\mathrm{a}, *}$, Dmitry IOFFE ${ }^{\mathrm{b}}$ \\ ${ }^{a}$ Dip. Matematica Universita di Roma Tre, L.go S. Murialdo 1, 00146 Roma, Italy \\ ${ }^{\mathrm{b}}$ Faculty of Industrial Engineering, Technion, Haifa 3200, Israel
}

Received 12 October 2001, revised 29 April 2002

\begin{abstract}
Consider uniformly elliptic random walk on $\mathbb{Z}^{d}$ with independent jump rates across nearest neighbour bonds of the lattice. We show that the infinite volume effective diffusion matrix can be almost surely recovered as the limit of finite volume periodized effective diffusion matrices.

(c) 2003 Éditions scientifiques et médicales Elsevier SAS

MSC: 60K37; 60K35; 74Q20

Keywords: Effective diffusion coefficient; Bond disorder; Corrector field

RÉSUMÉ. - Pour un modèle reversible et elliptique de marche aléatoire en milieu aléatoire indépendant on montre que le coefficient effectif de diffusion en volume infini peut être obtenu comme limite presque sûre des coefficients effectifs en volume fini.

() 2003 Éditions scientifiques et médicales Elsevier SAS
\end{abstract}

\section{Introduction}

Consider the following model of random walk in random environment: Given $c \geqslant 1$, the space of environments

$$
\Omega=\left\{\xi(b) \in[1 / c, c], \quad b \in \mathcal{E}^{d}\right\},
$$

is specified by the jump rates across the nearest neighbour bonds $b \in \mathcal{E}^{d}$, with $\mathcal{E}^{d}$ denoting the set of edges of the $d$-dimensional integer lattice $\mathbb{Z}^{d}$. We consider the product measure $\mu=\mu_{0}^{\otimes \mathcal{E}^{d}}$ on $\Omega$ obtained from a probability measure $\mu_{0}$ supported on $[1 / c, c]$.

\footnotetext{
Partly supported by the ISRAEL SCIENCE FOUNDATION founded by The Israel Academy of Science and Humanities.

* Corresponding author.

E-mail addresses: caputo@mat.uniroma3.it (P. Caputo), ieioffe@ie.technion.ac.il (D. Ioffe).
} 
For every fixed realization $\xi \in \Omega$ of the environment we denote $X(t, \xi)$ the nearest neighbour continuous time random walk on $\mathbb{Z}^{d}$ which starts at the origin and jumps according to $\xi(b)$ rates. The symbol $\mathbb{E}^{\xi}$ stands for expectation over this process. This is the quenched regime.

In the annealed regime the initial random environment is sampled from $\mu$. In the uniformly elliptic case we consider here the infinite volume annealed homogenization result is well known [14,11,10,3], and the effective diffusion matrix $D$ of $X(t)=X(t, \xi)$ is defined by

$$
\forall v \in \mathbb{R}^{d} \quad(D v, v)=\sum_{i, j=1}^{d} v_{i} D^{i j} v_{j}=\lim _{t \rightarrow \infty} \frac{1}{t} \mathbb{E}_{\mu} \mathbb{E}^{\xi}((X(t, \xi), v))^{2} .
$$

We shall briefly recall the Kipnis-Varadhan theory and the variational formula for $(D v, v)$ in Section 2.1. Meanwhile let us proceed with describing the finite volume approximation for the random environment. This is done in a straightforward fashion: Let $N \in \mathbb{Z}_{+}$and denote by $\pi_{N} \xi$ the periodized bond configuration

$$
\left(\pi_{N} \xi\right)_{i}(x)=\xi_{i}(\dot{x}), \quad x \in \mathbb{Z}^{d}, \dot{x} \in T_{N} \triangleq \mathbb{Z}^{d} / 2 N \mathbb{Z}^{d},
$$

where $\xi_{i}(x), i=1, \ldots, d$, stands for the rate $\xi(b)$ across the bond $b=\left(x, x+e_{i}\right)$, with $e_{i}$ the unit vector in the $i$ th direction. Here $\dot{x} \in\{-N, \ldots, N-1\}^{d}, x=\dot{x}+2 N z, z \in \mathbb{Z}^{d}$, and $\pi_{N} \xi$ is a periodic configuration which coincides with $\xi$ on the bonds belonging to the torus $T_{N}$. We consider the process $X\left(t, \pi_{N} \xi\right)$ describing a particle moving in $\mathbb{Z}^{d}$ according to the rates $\pi_{N} \xi$. As we shall recall in the beginning of Section 3, the finite volume homogenization result for $X\left(t, \pi_{N} \xi\right)$ is, actually, a much simpler statement than the infinite volume one. In particular, there is essentially no difference between the quenched and annealed regimes, and $\forall \xi \in \Omega$ the finite volume effective diffusion matrix $D_{N}(\xi)$ is well defined and given by

$$
\forall v \in \mathbb{R}^{d} \quad\left(D_{N}(\xi) v, v\right)=\sum_{i, j=1}^{d} v_{i} D_{N}^{i j}(\xi) v_{j}=\lim _{t \rightarrow \infty} \frac{1}{t} \mathbb{E}^{\pi_{N} \xi}\left(\left(X\left(t, \pi_{N} \xi\right), v\right)\right)^{2} .
$$

Notice that in general the value of $D_{N}=D_{N}(\xi)$ depends on the realization of the periodized environment $\pi_{N} \xi$.

Our main result here asserts that the finite volume effective diffusivities are rapidly self-averaging and, furthermore, the sequence $\left\{D_{N}(\xi)\right\}$ converges to the annealed infinite volume diffusivity $D$.

THEOREM 1.1. - There exists a power $v=v(c, d)>0$, such that:

$$
\max _{i, j} \mu\left(\left|D_{N}^{i j}(\xi)-\mathbb{E}_{\mu} D_{N}^{i j}(\xi)\right|>N^{-v}\right)<\mathrm{e}^{-N^{v}},
$$

for all $N$ sufficiently large. Furthermore,

$$
\lim _{N \rightarrow \infty} \mathbb{E}_{\mu} D_{N}(\xi)=D
$$


Remark 1. - As it has been pointed out by an anonymous referee it happens to be an easy matter to prove a complementary exponential lower bound: There exist $\varepsilon>0$ and $\kappa>0$ such that

$$
\max _{i, j} \mu\left(\left|D_{N}^{i j}(\xi)-\mathbb{E}_{\mu} D_{N}^{i j}(\xi)\right|>\varepsilon\right)>\mathrm{e}^{-\kappa N^{d}} .
$$

A sharp large deviation type description of $D_{N}(\xi)$ remains an open problem.

Of course, as an immediate consequence of Theorem 1.1 we obtain that $\mu$-almost surely,

$$
\lim _{N \rightarrow \infty} D_{N}(\xi)=D .
$$

Our proof of Theorem 1.1 strongly relies on specific properties of the environment measure $\mu$ : the concentration type result (1.3) relies on the independence of the jump rates $\xi(b)$, whereas the proof of (1.4) makes use of the exchangeability of the joint distribution of $\xi(b)$. Most recently the dual variational description of the inverse matrices $D_{N}^{-1}$ and $D^{-1}$ has been used to establish the convergence result (1.5) in the general context of ergodic jump rates [17]. The finite volume estimate (1.3) should, of course, require more stringent assumptions on the mixing properties of the $\xi$-field and so far (1.3) seems to be the only result in this direction. Also, as we shall explain in Section 4.5, our proof has an additional advantage of giving a natural interpretation of the translation covariant states for a harmonic interface in a random environment in terms of the FunakiSpohn states [5].

We would like to mention that approximation results of the type (1.4) or (1.5) go beyond the general spectral analysis of the Kipnis-Varadhan approach as developed in [10] or [3]. This issue is briefly addressed in Section 4.4. Similar approximation results have been recently derived in [12] in a much more delicate case of the self-diffusion coefficient of the tagged particle in the exclusion process.

Our main motivation for this work came from the theory of massless gradient fields on $\mathbb{Z}^{d}$. These are specified by the formal Hamiltonian

$$
\mathcal{H}(\phi)=\sum_{x} \sum_{i=1}^{d} U\left(\nabla_{i} \phi(x)\right)=\sum_{x} \sum_{i=1}^{d} U\left(\eta_{i}(x)\right),
$$

where $\nabla_{i}$ is the discrete lattice gradient; $\eta_{i}(x)=\nabla_{i} \phi(x)=\phi\left(x+e_{i}\right)-\phi(x)$. In the uniformly elliptic case, $1 / c \leqslant U^{\prime \prime}(\cdot) \leqslant c$, the infinite volume gradient states exist in any dimension $d \geqslant 1$, and, as it has been established in the paper by Funaki and Spohn [5], any translation invariant infinite volume gradient state is decomposable into the convex combination of the extremal states (the so called Funaki-Spohn states), which can be constructed as limits of finite volume periodized measures with appropriately tilted slopes. The very same approximation by the periodized states leads [5] to a meaningful definition of the (slope-dependent) surface tension. The latter is known to be strictly convex $[4,7]$.

On the mesoscopic level, the integral of the surface tension happens to be precisely the large deviation rate function for the appropriately scaled height field [4], and it has been conjectured in [7] that the Hessian of the surface tension governs the equilibrium 
fluctuations in the corresponding Funaki-Spohn state. In view of the random walk representation developed in [4], this conjecture would follow from an approximation result along the lines of Theorem 1.1, but for a different type of environment. We refer to [7] for further details. To the best of our knowledge this is still an open problem.

The paper is organized as follows: In Section 2 we briefly recall the variational formula for the infinite volume effective diffusion matrix and express it in terms of the corrector field. The relevant properties of the corrector field are listed in Proposition 2.1 and, other way around, we show that for every $v \in \mathbb{R}^{d}$ the corrector field $\psi_{v}$ is uniquely determined by these properties. Subsequently, $\psi_{v}$ is recovered as the $L^{2}(\mu)$ limit of the periodized corrector fields $\widetilde{\psi}_{N, v}$ in Proposition 3.1 of Section 3. This already leads to the convergence of the averaged finite volume effective diffusivities $\mathbb{E}_{\mu} D_{N}(\xi)$, Corollary 3.2. In Section 4 we show that $D_{N}(\xi)$ concentrates around its expected value $\mathbb{E}_{\mu} D_{N}(\xi)$. The concentration estimates of Section 4.3 prove the main claim in Theorem 1.1. The key ingredient of the proof is an a-priori $L^{p}$ estimate of Section 4.1, which is used to derive appropriate bounds on the Hamming distance in Section 4.2. By Meyers argument such $L^{p}$ estimate follows from discrete version of Calderon-Zygmund inequality. Since we could not find direct references to the latter, the proof is sketched in Appendix A.

\section{Diffusivity in the infinite medium}

\subsection{The variational formula}

We will denote $\xi_{i}(x), i=1, \ldots, d, x \in \mathbb{Z}^{d}$, the rate $\xi(b)$ at the bond $b=\left(x, x+e_{i}\right)$, with $e_{i}$ the unit vector in the $i$ th direction. When we write $\xi_{i}$ only we mean $\xi_{i}(0) . \tau_{y} \xi$ denotes the shifted configuration $\left(\tau_{y} \xi\right)_{i}(x)=\xi_{i}(x-y), x, y \in \mathbb{Z}^{d}, i=1, \ldots, d$. We also use the notation, for any $f: \Omega \rightarrow \mathbb{R}$,

$$
D_{i} f(\xi)=f\left(\tau_{-e_{i}} \xi\right)-f(\xi), \quad D_{i}^{*} f(\xi)=-D_{i} f\left(\tau_{e_{i}} \xi\right) .
$$

In the above notation the associated process of the environment $\xi_{t}=\tau_{X(t)} \xi$ seen from the particle (see [10,3]) is a Markov process with infinitesimal generator

$$
\mathcal{L} f(\xi)=-\sum_{i=1}^{d} D_{i}^{*}\left(\xi_{i} D_{i} f\right)(\xi) .
$$

By translation invariance $\mu$ is a reversible measure for this process and (2.1) defines a bounded self adjoint operator in $L^{2}(\mu)$ with $\mathbb{E}_{\mu}[g \mathcal{L} f]=-\sum_{i=1}^{d} \mathbb{E}_{\mu}\left[\xi_{i} D_{i} g D_{i} f\right]$, $f, g \in L^{2}(\mu)$.

It is well known [3] that the annealed effective diffusion matrix $D$ in (1.1) can be recovered from the variational formula:

$$
(D v, v)=2 \inf _{f \in L^{2}(\mu)} \sum_{i=1}^{d} \mathbb{E}_{\mu}\left(\xi_{i}\left(v_{i}+D_{i} f\right)^{2}\right) .
$$




\subsection{Corrector field}

In general, the above variational problem cannot be solved for $f$. However the next proposition shows that if we only look at "gradients" of $f$ the problem has a unique solution.

Proposition 2.1. - For every $v \in \mathbb{R}^{d}$ we have

$$
(D v, v)=2 \sum_{i=1}^{d} v_{i} \mathbb{E}_{\mu} \xi_{i}\left(v_{i}+\psi_{v}^{i}\right)=2 \sum_{i=1}^{d} \mathbb{E}_{\mu} \xi_{i}\left(v_{i}+\psi_{v}^{i}\right)^{2}
$$

where $\psi_{v}^{i}, i=1, \ldots, d$, are the unique elements of $L^{2}(\mu)$ such that

- $\mathbb{E}_{\mu} \psi_{v}^{i}=0$,

- $\sum_{i=1}^{d} D_{i}^{*}\left[\xi_{i}\left(v_{i}+\psi_{v}^{i}(\xi)\right)\right]=0, \mu$-a.s.,

- $D_{k} \psi_{v}^{i}=D_{i} \psi_{v}^{k}, \mu$-a.s., $i, k=1, \ldots, d$.

The above statement is well known and can be proven in several ways $[18,14,9]$. However, for the convenience of the reader and for the purpose of recalling relevant quantities in the Kipnis-Varadhan approach a proof is given below.

Proof. - For any vector $v \in \mathbb{R}^{d}$ we define the local drift or current along $v$ as

$$
\varphi_{v}(\xi)=\sum_{i=1}^{d} v_{i}\left(\xi_{i}(0)-\xi_{i}\left(-e_{i}\right)\right)=-\sum_{i=1}^{d} v_{i} D_{i}^{*} \xi_{i}(0) .
$$

Let us now define the functions

$$
\chi_{v}^{\lambda}=(-\mathcal{L}+\lambda)^{-1} \varphi_{v}, \quad \lambda>0, v \in \mathbb{R}^{d},
$$

or, in terms of the process:

$$
\chi_{v}^{\lambda}(\xi)=\mathbb{E}^{\xi} \int_{0}^{\infty} \mathrm{e}^{-\lambda t} \varphi_{v}\left(\tau_{X(t)} \xi\right) \mathrm{d} t
$$

Following Kipnis and Varadhan [10], Theorem 1.3 - see also [16] - one can use the spectral resolution of the non-negative operator $-\mathcal{L}$ on $L^{2}(\mu)$ to prove

$$
\lambda \mathbb{E}_{\mu}\left(\chi_{v}^{\lambda}\right)^{2} \rightarrow 0, \quad \lambda \rightarrow 0^{+} .
$$

Moreover one can prove that there exist functions $\psi_{v}^{i} \in L^{2}(\mu), i=1, \ldots, d$, such that

$$
\sum_{i=1}^{d} \mathbb{E}_{\mu} \xi_{i}\left(D_{i} \chi_{v}^{\lambda}-\psi_{v}^{i}\right)^{2} \rightarrow 0, \quad \lambda \rightarrow 0^{+}
$$


which in turn by the ellipticity of $\xi$ implies strong convergence of $D_{i} \chi_{v}^{\lambda}$ to $\psi_{v}^{i}$ in $L^{2}(\mu)$. In particular, we have

$$
\mathbb{E}_{\mu}\left(\varphi_{v}(-\mathcal{L})^{-1} \varphi_{v}\right)=\lim _{\lambda \rightarrow 0^{+}} \mathbb{E}_{\mu} \varphi_{v} \chi_{v}^{\lambda}=-\lim _{\lambda \rightarrow 0^{+}} \sum_{i=1}^{d} v_{i} \mathbb{E}_{\mu} \xi_{i} D_{i} \chi_{v}^{\lambda}=-\sum_{i=1}^{d} v_{i} \mathbb{E}_{\mu} \xi_{i} \psi_{v}^{i}
$$

Since (2.2) is equivalent to

$$
(D v, v)=2 \sum_{i=1}^{d} \mathbb{E}_{\mu}\left(\xi_{i}\right) v_{i}^{2}-2 \mathbb{E}_{\mu}\left(\varphi_{v}(-\mathcal{L})^{-1} \varphi_{v}\right),
$$

we obtain the first identity in (2.3).

To prove the second one we observe that

$$
\sum_{i=1}^{d} \mathbb{E}_{\mu} \xi_{i}\left(\psi_{v}^{i}\right)^{2}=-\sum_{i=1}^{d} v_{i} \mathbb{E}_{\mu} \xi_{i} \psi_{v}^{i}
$$

This follows from (2.6) and the convergence of $D_{i} \chi_{v}^{\lambda}$ to $\psi_{v}^{i}$ (2.7) since

$$
\begin{aligned}
\sum_{i=1}^{d} \mathbb{E}_{\mu} \xi_{i}\left(\psi_{v}^{i}\right)^{2} & =\lim _{\lambda \rightarrow 0^{+}} \sum_{i=1}^{d} \mathbb{E}_{\mu} \xi_{i}\left(D_{i} \chi_{v}^{\lambda}\right)^{2}=\lim _{\lambda \rightarrow 0^{+}} \mathbb{E}_{\mu}\left(\chi_{v}^{\lambda}(-\mathcal{L}) \chi_{v}^{\lambda}\right) \\
& =\lim _{\lambda \rightarrow 0^{+}}\left\{\mathbb{E}_{\mu} \chi_{v}^{\lambda} \varphi_{v}-\lambda \mathbb{E}_{\mu}\left(\chi_{v}^{\lambda}\right)^{2}\right\}=-\sum_{i=1}^{d} v_{i} \mathbb{E}_{\mu} \xi_{i} \psi_{v}^{i} .
\end{aligned}
$$

Next we show that the functions $\psi_{v}$ satisfy the three properties in the statement. The first property is obvious since $\psi_{v}^{i}$ is the $L^{2}(\mu)$-limit of a gradient and $\mu$ is translation invariant. To prove the second identity recall that, in view of (2.6)

$$
\lim _{\lambda \rightarrow 0^{+}} \mathbb{E}_{\mu}\left(f(-\mathcal{L}) \chi_{v}^{\lambda}\right)=\mathbb{E}_{\mu}\left(f \varphi_{v}\right), \quad f \in L^{2}(\mu) .
$$

Therefore for any $f \in L^{2}(\mu)$ we have

$$
\sum_{i=1}^{d} \mathbb{E}_{\mu} f D_{i}^{*}\left[\xi_{i}\left(v_{i}+\psi_{v}^{i}(\xi)\right)\right]=-\mathbb{E}_{\mu}\left(f \varphi_{v}\right)+\lim _{\lambda \rightarrow 0^{+}} \mathbb{E}_{\mu}\left(f(-\mathcal{L}) \chi_{v}^{\lambda}\right)=0 .
$$

The third identity follows in a similar fashion from $D_{k} D_{i} \chi_{v}^{\lambda}=D_{i} D_{k} \chi_{v}^{\lambda}, \lambda>0$.

Finally, to prove uniqueness we adapt the argument of Theorem 2 in [18] and of Proposition 3 in [2]: In the language of [2] the field $\psi_{v}(\xi)=\left(\psi_{v}^{1}(\xi), \ldots, \psi_{v}^{d}(\xi)\right)$ is, by the third of the properties in (2.3), an $L^{2}(\mu)$-cocycle. A straightforward modification of the proof of Proposition 3 in [2] reveals that any $L^{2}(\mu)$-cocycle $\left(u^{1}(\xi), \ldots, u^{d}(\xi)\right)$ is, in fact, an $L^{2}$-limit of gradients: There exists a sequence $g_{\gamma} \in L^{2}(\mu), \gamma>0$, with

$$
\mathbb{E}_{\mu}\left(D_{k} g_{\gamma}-u^{k}\right)^{2} \rightarrow 0, \quad \gamma \rightarrow 0^{+}, k=1, \ldots, d .
$$


Uniqueness of $\psi_{v}$ is an easy consequence. Indeed, fix $v$ and let $u^{k}=\psi_{1, v}^{k}-\psi_{2, v}^{k}$, with $\psi_{1, v}, \psi_{2, v}$ both satisfying the required conditions. We then have

$$
\sum_{k=1}^{d} \mathbb{E}_{\mu} \xi_{k} u^{k} D_{k} f=0, \quad f \in L^{2}(\mu) .
$$

Choosing $f=g_{\gamma}$ in (2.11), with $g_{\gamma}$ as in (2.10), we obtain

$$
\sum_{k=1}^{d} \mathbb{E}_{\mu} \xi_{k}\left(u^{k}\right)^{2}=\lim _{\gamma \rightarrow 0^{+}} \sum_{k=1}^{d} \mathbb{E}_{\mu} \xi_{k} u^{k} D_{k} g_{\gamma}=0,
$$

which, by the ellipticity of $\xi$, implies $u^{k}=0 \mu$-a.e., $k=1, \ldots, d$.

Remark 2. - Using the linearity in $v$ implicit in the definition (2.5) we may define the functions $\psi^{i j} \triangleq \psi_{e_{j}}^{i}, i, j=1, \ldots, d$, so that

$$
\psi_{v}^{i}=(\Psi v)_{i} \triangleq \sum_{j=1}^{d} \psi^{i j} v_{j} .
$$

The diffusivity $D$ is then given by the matrix

$$
D=2 \mathbb{E}_{\mu} \Lambda(1+\Psi)
$$

with $\Lambda$ denoting the diagonal matrix $\Lambda^{i j}=\xi_{i} \delta_{i j}$. The functions $\psi^{i j}$ are often called the corrector fields and $\Psi$ the stream matrix.

Remark 3. - The diffusion constant $D$ has an explicit expression if $d=1$. Indeed, for any $v \in \mathbb{R}, \xi\left(v+\psi_{v}(\xi)\right)$ must be constant $\mu$-a.s. and normalizing we have

$$
\psi_{v}(\xi)=v\left[\xi^{-1}\left(\mathbb{E}_{\mu} \xi^{-1}\right)^{-1}-1\right], \quad D=\left(\mathbb{E}_{\mu} \xi^{-1}\right)^{-1} .
$$

By ellipticity we see that the corrector field $\psi_{v}$ is uniformly bounded in this case. The situation is different if $d \geqslant 2$. There is no longer an expression for $\psi_{v}$ and all we know apriori is that $\psi \in L^{2}(\mu)$. For $d=2$ this has been upgraded in [1] to $\psi \in L^{2+\varepsilon}(\mu)$ for some $\varepsilon>0$, which (in two dimensions) leads to an almost sure homogenization result. In the sequel, c.f. the remark following Theorem 4.1, we shall establish that $\psi \in L^{2+\varepsilon}(\mu)$ in any dimension $d \geqslant 2$.

\section{Diffusivity in the periodic medium}

In the case of the periodized environment (1.2), we consider the projected random walk $\dot{X}\left(t, \pi_{N} \xi\right)$ on $T_{N}$, i.e. an irreducible Markov chain with finite state space and unique invariant measure $\rho_{N}$ defined by

$$
\mathbb{E}_{\rho_{N}} f=\frac{1}{\left|T_{N}\right|} \sum_{x \in T_{N}} f(x), \quad f: T_{N} \rightarrow \mathbb{R} .
$$


Then, introducing the periodic function

$$
\varphi_{v}^{N}(x)=\varphi_{v}\left(\tau_{-x} \pi_{N} \xi\right)
$$

where $\varphi_{v}$ is defined by (2.4), we arrive as in (2.8) at the expression

$$
\left(D_{N}(\xi) v, v\right)=2 \sum_{i=1}^{d} \mathbb{E}_{\rho_{N}}\left(\left(\pi_{N} \xi\right)_{i}\right) v_{i}^{2}-2 \mathbb{E}_{\rho_{N}}\left(\varphi_{v}^{N}\left(-\mathcal{L}_{N}\right)^{-1} \varphi_{v}^{N}\right)
$$

where the generator $\mathcal{L}_{N}$ describes the jumps on $T_{N}$, i.e. for any periodic function $f$ on $\mathbb{Z}^{d}$

$$
\mathcal{L}_{N} f(x)=\left(\pi_{N} \xi\right)_{i}(x)\left[f\left(x+e_{i}\right)-f(x)\right]+\left(\pi_{N} \xi\right)_{i}\left(x-e_{i}\right)\left[f\left(x-e_{i}\right)-f(x)\right] .
$$

Alternatively, identifying periodic functions with functions on $T_{N}$ we may write

$$
\mathcal{L}_{N} f(x)=-\sum_{i=1}^{d} \widetilde{\nabla}_{i}^{*}\left(\left(\pi_{N} \xi\right)_{i}(x) \widetilde{\nabla}_{i} f(x)\right), \quad f: T_{N} \rightarrow \mathbb{R},
$$

where $\widetilde{\nabla}$ are the discrete gradients on the torus $T_{N}$. We then have the variational principle

$$
\left(v, D_{N}(\xi) v\right)=2 \inf _{f: T_{N} \rightarrow \mathbb{R}} \sum_{i=1}^{d} \mathbb{E}_{\rho_{N}}\left(\left(\pi_{N} \xi\right)_{i}\left(v_{i}+\widetilde{\nabla}_{i} f\right)^{2}\right) .
$$

In this finite-dimensional setting the above problem may be solved directly without the approximation procedure outlined in the proof of Proposition 2.1. Namely, define

$$
\chi_{N, v}(x)=\left(-\mathcal{L}_{N}\right)^{-1} \varphi_{v}^{N}(x), \quad x \in \mathbb{Z}^{d},
$$

and observe that $\chi_{N, v}$ is a bounded periodic function for each $N$. Boundedness follows from the exponential mixing properties of the process generated by $\mathcal{L}_{N}$, since $\mathbb{E}_{\rho_{N}} \varphi_{v}^{N}=$ 0 . As in the previous section we then have

$$
\left(v, D_{N}(\xi) v\right)=2 \sum_{i=1}^{d} v_{i} \mathbb{E}_{\rho_{N}}\left(\pi_{N} \xi\right)_{i}\left(v_{i}+\psi_{N, v}^{i}\right)=2 \sum_{i=1}^{d} \mathbb{E}_{\rho_{N}}\left(\pi_{N} \xi\right)_{i}\left(v_{i}+\psi_{N, v}^{i}\right)^{2}
$$

where $\psi_{N, v}^{i}, i=1, \ldots, d$, are defined by

$$
\psi_{N, v}^{i}(x)=\chi_{N, v}\left(x+e_{i}\right)-\chi_{N, v}(x)=\widetilde{\nabla}_{i} \chi_{N, v}(x) .
$$

Clearly $\psi_{N, v}^{i}$ are periodic functions. The dependence on $\xi$ becomes explicit in the notation

$$
\psi_{N, v}^{i}(x, \xi)=\psi_{N, v}^{i}(x)=\psi_{N, v}^{i}\left(0, \tau_{-x} \pi_{N} \xi\right)
$$




\subsection{Convergence}

From now on $\psi_{N, v}^{i}$ will be regarded as a function of $\xi$, by setting

$$
\widetilde{\psi}_{N, v}^{i}(\xi)=\psi_{N, v}^{i}(0, \xi), \quad i=1, \ldots, d .
$$

Proposition 3.1. - For each $v \in \mathbb{R}^{d}, \widetilde{\psi}_{N, v} \rightarrow \psi_{v}$ in $L^{2}(\mu)$, as $N \rightarrow \infty$, where $\psi_{v}$ is the field defined in Proposition 2.1.

Proof. - Notice that, as in (2.9)

$$
\sum_{i=1}^{d} \mathbb{E}_{\rho_{N}}\left(\pi_{N} \xi\right)_{i}\left(\psi_{N, v}^{i}\right)^{2}=-\sum_{i=1}^{d} v_{i} \mathbb{E}_{\rho_{N}}\left(\pi_{N} \xi\right)_{i} \psi_{N, v}^{i}
$$

Then by ellipticity and using Schwarz' inequality we obtain

$$
\max _{i} \mathbb{E}_{\rho_{N}}\left(\psi_{N, v}^{i}\right)^{2} \leqslant c^{2}|v|^{2} .
$$

Now we observe that since the bond variables $\xi$ are exchangeable under $\mu$, the distribution of $\pi_{N} \xi$ coincides with that of $\tau_{x} \pi_{N} \xi$ for any $x \in \mathbb{Z}^{d}$. In particular we see that

$$
\mathbb{E}_{\mu}\left(\widetilde{\psi}_{N, v}^{i}\right)^{2}=\frac{1}{\left|T_{N}\right|} \sum_{x \in T_{N}} \mathbb{E}_{\mu}\left(\psi_{N, v}^{i}\left(0, \tau_{x} \pi_{N} \xi\right)\right)^{2}=\mathbb{E}_{\mu} \mathbb{E}_{\rho_{N}}\left(\psi_{N, v}^{i}(\cdot, \xi)\right)^{2}
$$

and (3.10) provides a uniform bound on the $L^{2}(\mu)$-norm of $\widetilde{\psi}_{N, v}^{i}$, which implies weak convergence along subsequences. Next we show that any weak limit point must satisfy the conditions of Proposition 2.1, thus establishing the weak convergence of $\widetilde{\psi}_{N, v}$ to $\psi_{v}$. Let $\widetilde{\psi}_{\infty, v}^{i}$ be a weak limit of $\widetilde{\psi}_{N, v}^{i} . \mathbb{E}_{\mu} \widetilde{\psi}_{\infty, v}^{i}=0$ is a consequence of $\mathbb{E}_{\mu} \widetilde{\psi}_{N, v}^{i}=0$, which in turn follows from exchangeability. To check the second condition we show that for any local function $f \in L^{2}(\Omega)$ we have

$$
\sum_{i=1}^{d} \mathbb{E}_{\mu}\left[D_{i} f \xi_{i}\left(v_{i}+\widetilde{\psi}_{\infty, v}^{i}(\xi)\right)\right]=0
$$

From the definition of $\psi_{N, v}^{i}$, writing out (3.5) explicitly we have

$$
\sum_{i=1}^{d} \widetilde{\nabla}_{i}^{*}\left(\left(\pi_{N} \xi\right)_{i}(x) \psi_{N, v}^{i}(x, \xi)\right)=\varphi_{v}^{N}(x), \quad x \in T_{N} .
$$

At $x=0$ this yields

$$
\sum_{i=1}^{d}\left[\left(\tau_{e_{i}} \xi\right)_{i}\left(v_{i}+\widetilde{\psi}_{N, v}^{i}\left(\tau_{e_{i}} \pi_{N} \xi\right)\right)-\xi_{i}\left(v_{i}+\widetilde{\psi}_{N, v}^{i}\left(\pi_{N} \xi\right)\right)\right]=0 .
$$


If $f \in L^{2}(\Omega)$ is local, then $f(\xi)=f\left(\pi_{N} \xi\right)$ and $f\left(\tau_{ \pm e_{j}} \xi\right)=f\left(\tau_{ \pm e_{j}} \pi_{N} \xi\right)$ when $N$ is large, and by the exchangeability we have

$$
\int_{\Omega} \mu(\mathrm{d} \xi) f\left(\pi_{N} \xi\right)\left(\tau_{e_{i}} \xi\right)_{i} \widetilde{\psi}_{N, v}^{i}\left(\tau_{e_{i}} \pi_{N} \xi\right)=\int_{\Omega} \mu(\mathrm{d} \xi) f\left(\tau_{-e_{i}} \pi_{N} \xi\right) \xi_{i} \widetilde{\psi}_{N, v}^{i}(\xi) .
$$

Multiplying (3.13) with $f$ and integrating we see that when $N$ is large

$$
\sum_{i=1}^{d} \mathbb{E}_{\mu}\left[D_{i} f \xi_{i}\left(v_{i}+\widetilde{\psi}_{N, v}^{i}\right)\right]=0,
$$

and the claim (3.12) follows from weak convergence. The last condition in Proposition 2.1 is proven by similar reasoning, using also

$$
\widetilde{\nabla}_{i} \psi_{N, v}^{k}=\widetilde{\nabla}_{i} \widetilde{\nabla}_{k} \chi_{N, v}=\widetilde{\nabla}_{k} \widetilde{\nabla}_{i} \chi_{N, v}=\widetilde{\nabla}_{k} \psi_{N, v}^{i} .
$$

By the orthogonality relations (2.9) and (3.9) we, using again exchangeability as in (3.11), infer:

$$
\lim _{N \rightarrow \infty} \sum_{i=1}^{d} \mathbb{E}_{\mu} \xi_{i}\left(\widetilde{\psi}_{N, v}^{i}\right)^{2}=\sum_{i=1}^{d} \mathbb{E}_{\mu} \xi_{i}\left(\psi_{v}^{i}\right)^{2}
$$

In view of the uniform ellipticity condition, $\xi_{i} \geqslant 1 / c>0$, this implies that $\widetilde{\psi}_{N, v}^{i}$ converge strongly in $L^{2}(\mu)$.

An immediate corollary of the above results is the mean convergence of the diffusion constants $\left\{D_{N}(\xi)\right\}$ :

COROLlary 3.2. - For every $v \in \mathbb{R}^{d}$,

$$
\lim _{N \rightarrow \infty} \mathbb{E}_{\mu}\left(v, D_{N}(\xi) v\right)=(v, D v)
$$

Proof. - Follows from the representation formulas (2.3) and (3.6), exchangeability and the $L^{2}(\mu)$ convergence of $\widetilde{\psi}_{N, v}^{i}$ to $\psi_{v}^{i}$.

\section{Almost sure convergence of $D_{N}$}

In this section we show that the periodized finite volume effective diffusion matrices $D_{N}(\xi)$ converge to the infinite volume effective diffusivity $D$ almost surely in the environment, that is (1.5) holds. $L^{p}$ bounds on the gradients $\widetilde{\nabla} \chi_{N, v}$ of the solutions to (3.5) set up stage for an application of Talagrand's concentration estimates, which imply that for every $v \in \mathbb{R}^{d}$ :

$$
\mu \text {-a.s. } \quad \lim _{N \rightarrow \infty}\left|\left(D_{N}(\xi) v, v\right)-\mathbb{E}_{\mu}\left(D_{N}(\xi) v, v\right)\right|=0 .
$$


In the sequel we shall use $\chi_{N, v}^{\xi}$ instead of $\chi_{N, v}$, just in order to stress the dependence of solutions of (3.5) on a particular realization

$$
\xi \in \Omega_{N} \triangleq[1 / c, c]^{T_{N}}
$$

of the periodized environment.

\section{1. $L^{p}$ estimates}

Eq. (3.5) is uniformly elliptic in $N \in \mathbb{N}$ and $\xi \in \Omega_{N}$.

THEOREM 4.1. - There exists a power $p=p(c)>2$ and a constant $\alpha=\alpha(c)<\infty$, such that uniformly in $v \in \mathbb{R}^{d}, N \in \mathbb{N}$ and $\xi \in \Omega_{N}$,

$$
\left\|\widetilde{\nabla} \chi_{N, v}^{\xi}\right\|_{N, p}=\left(\frac{1}{\left|T_{N}\right|} \sum_{x \in T_{N}}\left|\widetilde{\nabla} \chi_{N, v}^{\xi}(x)\right|^{p}\right)^{1 / p} \leqslant \alpha|v| .
$$

Remark. - By the exchangeability of the $\xi$-environment, (4.2) implies

$$
\sup _{N} \mathbb{E}_{\mu}\left|\widetilde{\psi}_{N, v}\right|^{p}<\infty,
$$

where $\widetilde{\psi}_{N, v}$ has been defined in (3.8). Since the infinite volume corrector field could be obtained as an almost sure limit of $\widetilde{\psi}_{N_{k}, v}$ along a subsequence $\left\{N_{k}\right\}$, it follows that $\psi_{v} \in L^{p}(\mu)$ for every $v \in \mathbb{R}^{d}$.

Because of the uniform ellipticity of $\mathcal{L}_{N}$ the inequality (4.2) follows by Meyers argument [15] (see also Step 1 in Section 3.3 in [4] for the discrete case) once the corresponding $L^{q}$ estimate holds for the Poisson equation

$$
\sum_{i=1}^{d} \widetilde{\nabla}_{i}^{*} \widetilde{\nabla}_{i} u(x)=-\sum_{i=1}^{d} \widetilde{\nabla}_{i}^{*} f_{i}(x)
$$

for some $q>2$. Namely, we have to show that there exists $\beta=\beta(q)<\infty$, such that for any $N \in \mathbb{N}$ and every vector field $\vec{f}=\left(f_{1}, \ldots, f_{d}\right)$ on $T_{N}$ solutions $u$ of (4.3) satisfy:

$$
\|\widetilde{\nabla} u\|_{N, q}^{q}=\frac{1}{\left|T_{N}\right|} \sum_{x \in T_{N}}|\widetilde{\nabla} u(x)|^{q} \leqslant \beta(q)\|\vec{f}\|_{N, q}^{q} .
$$

(4.4) is a discrete version of Calderon-Zygmund inequality. We could not find a direct reference which would cover the case we consider here. For the convenience of the reader, a brief sketch of the proof is given in Appendix A.

\subsection{Bounds on the Hamming distance}

For $\xi, \xi^{\prime} \in \Omega_{N}$ let us define the Hamming distance

$$
\mathrm{d}_{\text {Ham }, N}\left\{\xi, \xi^{\prime}\right\}=\sum_{x \in T_{N}} \sum_{i=1}^{d} \delta_{\xi_{i}(x) \neq \xi_{i}^{\prime}(x)} .
$$


Set $q=p /(p-2)<\infty$, where $p=p(c)>2$ is as in the statement on Theorem 4.1. We claim that uniformly in $v$ and in $\xi, \xi^{\prime} \in \Omega_{N}$,

$$
\left|\left(D_{N}(\xi) v, v\right)-\left(D_{N}\left(\xi^{\prime}\right) v, v\right)\right| \leqslant c_{1}|v|^{2}\left[\frac{1}{\left|T_{N}\right|} \mathrm{d}_{\mathrm{Ham}, N}\left\{\xi, \xi^{\prime}\right\}\right]^{1 / q} .
$$

Indeed, by (3.4) and (3.6),

$$
\begin{aligned}
\frac{1}{2}\left(D_{N}(\xi) v, v\right) & =\min _{f: T_{N} \mapsto \mathbb{R}} \frac{1}{\left|T_{N}\right|} \sum_{x \in T_{N}} \sum_{i=1}^{d} \xi_{i}(x)\left(v_{i}+\widetilde{\nabla}_{i} f\right)^{2} \\
& =\frac{1}{\left|T_{N}\right|} \sum_{x \in T_{N}} \sum_{i=1}^{d} \xi_{i}(x)\left(v_{i}+\widetilde{\nabla}_{i} \chi_{N, v}^{\xi}\right)^{2},
\end{aligned}
$$

for every $v \in \mathbb{R}^{d}$ and $\xi \in \Omega_{N}$. Consequently, in view of the a-priori estimate (4.2), we infer:

$$
\begin{aligned}
& \frac{1}{2}\left|\left(D_{N}(\xi) v, v\right)-\left(D_{N}\left(\xi^{\prime}\right) v, v\right)\right| \\
& \quad \leqslant \frac{1}{\left|T_{N}\right|} \sum_{x \in T_{N}} \sum_{i=1}^{d}\left|\xi_{i}(x)-\xi_{i}^{\prime}(x)\right|\left[\left(v_{i}+\widetilde{\nabla}_{i} \chi_{N, v}^{\xi}(x)\right)^{2}+\left(v_{i}+\widetilde{\nabla}_{i} \chi_{N, v}^{\xi^{\prime}}(x)\right)^{2}\right] \\
& \quad \leqslant c_{2}|v|^{2}\left[\frac{1}{\left|T_{N}\right|} \sum_{x \in T_{N}} \sum_{i=1}^{d}\left|\xi_{i}(x)-\xi_{i}^{\prime}(x)\right|^{q}\right]^{1 / q} .
\end{aligned}
$$

Obviously,

$$
\sum_{x \in T_{N}} \sum_{i=1}^{d}\left|\xi_{i}(x)-\xi_{i}^{\prime}(x)\right|^{q} \leqslant c^{q} \mathrm{~d}_{\mathrm{Ham}, N}\left\{\xi, \xi^{\prime}\right\},
$$

uniformly in $\xi, \xi^{\prime} \in \Omega_{N}$, and the bound (4.5) follows.

\subsection{Concentration of $D_{N}(\xi)$}

By (4.6)

$$
0 \leqslant\left(D_{N}(\xi) v, v\right) \leqslant c|v|^{2}
$$

for every $v \in \mathbb{R}^{d}$ and for each realization of the environment $\xi \in \Omega_{N}$. Thus, given $\varepsilon_{N}>0$ one can find a value $\bar{D}_{N}(v) \in\left[0, c|v|^{2}\right]$, such that $\mu\left(A_{N}(v)\right) \geqslant 2 \varepsilon_{N} /\left(c|v|^{2}\right)$, where we define the set $A_{N}(v) \subset \Omega_{N}$ as:

$$
A_{N}(v)=\left\{\xi \in \Omega_{N}:\left|\left(D_{N}(\xi) v, v\right)-\bar{D}_{N}(v)\right|<\varepsilon_{N}\right\} .
$$

Every $\xi^{\prime} \in \Omega_{N}$ such that

$$
\left|\left(D_{N}\left(\xi^{\prime}\right) v, v\right)-\bar{D}_{N}(v)\right|>2 \varepsilon_{N},
$$


certainly satisfies

$$
\min _{\xi \in A_{N}(v)}\left|\left(D_{N}(\xi) v, v\right)-\left(D_{N}\left(\xi^{\prime}\right) v, v\right)\right|>\varepsilon_{N} .
$$

By (4.5) we arrive to the following bound on such $\xi^{\prime}$ in terms of the Hamming distance:

$$
\mathrm{d}_{\text {Ham }, N}\left\{\xi^{\prime}, A_{N}(v)\right\}=\min _{\xi \in A_{N}(v)} \mathrm{d}_{\text {Ham }, N}\left\{\xi^{\prime}, \xi\right\}>c_{4}\left(\frac{\varepsilon_{N}}{|v|^{2}}\right)^{q} N^{d} .
$$

We are now in position to use the concentration estimate (5.2) in [19]:

$$
\begin{aligned}
& \mu\left(\left|\left(D_{N}\left(\xi^{\prime}\right) v, v\right)-\bar{D}_{N}(v)\right|>2 \varepsilon_{N}\right) \\
& \quad \leqslant \mu\left(\xi^{\prime}: \mathrm{d}_{\text {Ham }, N}\left\{\xi^{\prime}, A_{N}(v)\right\}>c_{4}\left(\frac{\varepsilon_{N}}{|v|^{2}}\right)^{q} N^{d}\right) \\
& \quad \leqslant \frac{1}{\mu\left(A_{N}(v)\right)} \exp \left\{-c_{5} \frac{\varepsilon_{N}^{2 q}}{|v|^{4 q}} N^{d}\right\} \leqslant \frac{c|v|^{2}}{2 \varepsilon_{N}} \exp \left\{-c_{6}(|v|) \varepsilon_{N}^{2 q} N^{d}\right\} .
\end{aligned}
$$

It remains to choose $\varepsilon_{N}=\sqrt{N^{-(d-\Delta) / q}}$ (consequently, $\varepsilon_{N}^{2 q} N^{d}=N^{\Delta}$ in (4.7) above) for some $\Delta>0$ : Since the sequence of random variables $\left(D_{N}(\xi) v, v\right)$ is uniformly bounded, we readily infer from (4.7) that

$$
\left|\mathbb{E}_{\mu}\left(D_{N}(\xi) v, v\right)-\bar{D}_{N}(v)\right|<3 \varepsilon_{N}
$$

for all large enough values of $N$, and the assertion (1.3) of Theorem 1.1 follows with $v=\min \{\Delta,(d-\Delta) / 2 q\}$.

\subsection{Spectral measures of the local drift $\varphi_{v}$}

In the notation of Section 3 let us introduce the empirical measures

$$
\mu_{N}=\mu_{N}^{\xi}=\frac{1}{\left|T_{N}\right|} \sum_{x \in T_{N}} \delta_{\tau_{-x} \pi_{N} \xi}
$$

Notice that for every $\xi \in \Omega$ fixed or, equivalently, for each $\pi_{N} \xi \in \Omega_{N}$ fixed there is an obvious correspondence between the spaces $L^{2}\left(T_{N}, \rho_{N}\right)$ and $L^{2}\left(\Omega_{N}, \mu_{N}\right)$ : for every $f \in L^{2}\left(T_{N}, \rho_{N}\right)$ define $\hat{f} \in L^{2}\left(\Omega_{N}, \mu_{N}\right)$ via $\hat{f}\left(\tau_{-x} \pi_{N} \xi\right)=f(x)$ and vice versa.

By (2.8) and (3.2) the limiting relation (1.5) could be written as

$$
\mu \text {-a.s. } \lim _{N \rightarrow \infty} \mathbb{E}_{\mu_{N}}\left(\varphi_{v}\left(-\mathcal{L}_{N}\right)^{-1} \varphi_{v}\right)=\mathbb{E}_{\mu}\left(\varphi_{v}(-\mathcal{L})^{-1} \varphi_{v}\right) .
$$

Fix now $v \in \mathbb{R}^{d}$. Let $v$ and, respectively, $v_{N}=v_{N}^{\xi}$ be the spectral measures of $\varphi_{v}$ relative to the operator $(-\mathcal{L})$ on $L^{2}(\Omega, \mu)$ and, respectively, relative to the operator $\left(-\mathcal{L}_{N}\right)$ on $L^{2}\left(\Omega_{N}, \mu_{N}^{\xi}\right)$. Both $-\mathcal{L}$ and all of $-\mathcal{L}_{N}$ are self-adjoint and bounded on the respective spaces. Let $K$ be a common upper bound on the spectral radiuses. In terms of spectral 
measures the limit in (4.8) reads as

$$
\mu \text {-a.s. } \lim _{N \rightarrow \infty} \int_{0}^{K} \frac{v_{N}^{\xi}(\mathrm{d} r)}{r}=\int_{0}^{K} \frac{\nu(\mathrm{d} r)}{r} .
$$

Kipnis-Varadhan approach is based on the fact that $\int_{0}^{K} \frac{v(\mathrm{~d} r)}{r}$ (or, respectively, $\int_{0}^{K} \frac{v_{N}^{\xi}(\mathrm{d} r)}{r}$ in the case of the periodized environment) is bounded above. We claim that our convergence result (4.9) is equivalent to the following uniform continuity near zero type property of the family $\left\{v_{N}^{\xi}(\mathrm{d} r) / r\right\}: \mu$-almost surely

$$
\lim _{\delta \rightarrow 0} \sup _{N} \int_{0}^{\delta} v_{N}^{\xi}(\mathrm{d} r) / r=0 .
$$

Such an equivalence would follow if, for example, we are able to show that $\mu$-almost surely the sequence $\left\{v_{N}^{\xi}\right\} *$-weakly converges to $\nu$. The latter is a consequence of

LEMMA 4.2.-

$$
\lim _{N \rightarrow \infty} \int_{0}^{K} \mathrm{e}^{-n r} v_{N}^{\xi}(\mathrm{d} r)=\int_{0}^{K} \mathrm{e}^{-n r} v(\mathrm{~d} r),
$$

$\mu$-a.s. for all $n \in \mathbb{N}$.

Proof. - The claim of the lemma essentially follows from the strong law of large numbers: Since the local drift $\varphi_{v}$ is bounded,

$$
\begin{aligned}
\int_{0}^{K} \mathrm{e}^{-n r} v_{N}^{\xi}(\mathrm{d} r)= & \frac{1}{[2(N-M)]^{d}} \sum_{x \in\{-N+M, \ldots, N-M-1\}^{d}} \varphi_{v}\left(\tau_{-x} \xi\right) \mathbb{E}_{x}^{\xi} \varphi_{v}^{N}\left(\dot{X}\left(n, \pi_{N} \xi\right)\right) \\
& +\mathrm{O}\left(\frac{M}{N}\right)
\end{aligned}
$$

where $\dot{X}\left(n, \pi_{N} \xi\right)$ is the wrapping round the torus $T_{N}$ of the random walk $X\left(n, \pi_{N} \xi\right)$ moving in the periodic environment $\pi_{N} \xi$ and $\mathbb{P}_{x}^{\xi}$ (resp. $\mathbb{E}_{x}^{\xi}$ ) denotes the law (resp. expectation) of such random walk with the starting point $\dot{X}\left(0, \pi_{N} \xi\right)=x$. Since the jump rates of $X(t, \xi)$ are, uniformly in $\xi \in \Omega$, bounded above by $c$,

$$
\max _{x \in T_{N}} \sup _{\xi \in \Omega} \mathbb{P}_{x}^{\xi}\left(\max _{0 \leqslant t \leqslant n}|X(t, \xi)-x|>M\right) \leqslant c_{5} \mathrm{e}^{-c_{6} M^{2} / n} .
$$

Consequently,

$$
\mathbb{E}_{x}^{\xi} \varphi_{v}^{N}\left(\dot{X}\left(n, \pi_{N} \xi\right)\right)=\mathbb{E}_{x}^{\xi} \varphi_{v}(X(n, \xi))+\mathrm{O}\left(\mathrm{e}^{-c_{6} M^{2} / n}\right),
$$

uniformly in $x \in\{-N+M, \ldots, N-M-1\}^{d}$. Substituting (4.12) into (4.11) and choosing $M=M(N)=\sqrt{N}$, we arrive to the claim of the lemma. 


\subsection{Some remarks on a massless Gaussian field with bond disorder}

A harmonic interface in the quenched random environment $\xi \in \Omega$ is described by the formal Hamiltonian

$$
\mathcal{H}(\phi)=\frac{1}{2} \sum_{x} \sum_{i=1}^{d} \xi_{i}(x)\left(\nabla_{i} \phi(x)\right)^{2},
$$

where $\nabla_{i} \phi(x)=\phi\left(x+e_{i}\right)-\phi(x)$ is the discrete gradient in $\mathbb{Z}^{d}$. We shall see below how the corrector fields introduced in previous sections can be used to characterize an interesting class of Gibbs measures for the interaction (4.13).

In order to localize the interface in $d=1,2$ we may consider the field pinned at the origin, i.e. we impose $\phi(0)=0$. Since the interaction (4.13) is quadratic a full description of the set $\mathcal{G}^{\xi}$ of infinite-volume Gibbs measures for a given typical realization $\xi \in \Omega$ is available. Namely, it is well known ([6], Theorems (13.22) and (13.24)) that the set of extremal elements of $\mathcal{G}^{\xi}$, denoted ext $\mathcal{G}^{\xi}$, coincides with the set of Gaussian fields on $\mathbb{R}^{\mathbb{Z}^{d}}$ with a $\xi$-harmonic mean vector $\chi: \mathbb{Z}^{d} \rightarrow \mathbb{R}$ satisfying

$$
\sum_{i} \nabla_{i}^{*}\left(\xi_{i}(x) \nabla_{i} \chi(x)\right)=0, \quad x \in \mathbb{Z}^{d} ; \chi(0)=0,
$$

and covariance $G^{\xi}(x, y)$ given by the Green function of the random walk in the $\xi$ environment killed upon hitting the origin. Here $\nabla_{i}^{*}$ denotes the conjugate gradient in $\mathbb{Z}^{d}: \nabla_{i}^{*} \phi(x)=\phi\left(x-e_{i}\right)-\phi(x)$. ext $\mathcal{G}^{\xi}$ is thus characterized by solutions to (4.14).

To study the tilted states associated to (4.13) it is convenient to work directly with the gradient field $\eta_{i} \triangleq \nabla_{i} \phi$. This is no loss of information in view of the condition $\phi(0)=0$. In particular, with the correspondence $\sigma_{i}=\nabla_{i} \chi,(4.14)$ is equivalent to

$$
\sum_{i} \nabla_{i}^{*}\left(\xi_{i}(x) \sigma_{i}(x)\right)=0, \quad \nabla_{k} \sigma_{i}(x)=\nabla_{i} \sigma_{k}(x), \quad i, k=1, \ldots, d, x \in \mathbb{Z}^{d},
$$

and $\operatorname{ext} \mathcal{G}^{\xi}$ is characterized by solutions to (4.15), so that $v \in \operatorname{ext} \mathcal{G}^{\xi}$ is a Gaussian measure on $\left(\mathbb{R}^{d}\right)^{\mathbb{Z}^{d}}$ with mean $\sigma$ satisfying (4.15) and covariance

$$
\begin{aligned}
C_{i j}^{\xi}(x, y) & \triangleq \operatorname{cov}_{v}\left(\eta_{i}(x), \eta_{j}(y)\right) \\
& =G^{\xi}\left(x+e_{i}, y+e_{j}\right)+G^{\xi}(x, y)-G^{\xi}\left(x+e_{i}, y\right)-G^{\xi}\left(x, y+e_{j}\right) .
\end{aligned}
$$

Translation covariance and tilted states. A random Gibbs measure is a measurable map $v: \Omega \rightarrow\left\{\mathcal{G}^{\xi}, \xi \in \Omega\right\}$ such that $v^{\xi} \in \mathcal{G}^{\xi}$ for every $\xi \in \Omega$. The map $v^{\prime}$ is called translation covariant when $\nu^{\xi} \circ \theta_{x}=v^{\tau_{x} \xi}$ for every $\xi \in \Omega, x \in \mathbb{Z}^{d}$, where $\theta_{x}$ denotes the action of translation group and $\tau_{x}$ is the environment shift. We define the tilted states for our interface in random environment as the set of translation covariant random Gibbs measures $v^{\prime}$ such that $v^{\xi} \in \operatorname{ext} \mathcal{G}^{\xi}$. It is not difficult now to use Proposition 2.1 to give a full characterization of tilted states. Indeed, since $C_{i j}^{\xi}$ is translation covariant, we only have to characterize the translation covariant maps $\xi \rightarrow \sigma^{\xi}$ with $\sigma^{\xi}$ obeying (4.15). For 
every $v \in \mathbb{R}^{d}$ we know that

$$
\sigma_{v}^{\xi}(x) \triangleq v+\psi_{v}\left(\tau_{-x} \xi\right)
$$

satisfies (4.15). On the other hand if we additionally require that $\sigma^{\xi}(0)$ is in $L^{2}(\Omega, \mu)$ then the uniqueness statement in Proposition 2.1 allows to conclude that $\sigma_{v}^{\xi}, v \in \mathbb{R}^{d}$ are the only maps with these properties. In other words, for every $v \in \mathbb{R}^{d}$ the Gaussian measure $\nu_{v}^{\xi}$ with covariance $C^{\xi}$ and mean $\sigma_{v}^{\xi}$ is the unique tilted state such that $\mathbb{E}_{\mu}\left[v_{v}^{\xi}(\eta(0))\right]=v$ and $\mathbb{E}_{\mu}\left[v_{v}^{\xi}\left(|\eta(0)|^{2}\right)\right]<\infty$. The above measures are the analogue in our setting of Funaki-Spohn states for translation invariant massless gradient fields ([5]).

Tilted states on the torus and surface tension. To carry this analogy a little further here we mimic the construction of [5] to describe tilted states as the infinite volume limit of tilted measure on the torus $T_{N}$.

Define $\Gamma_{N}$ as the set of $\tilde{\eta} \in\left(\mathbb{R}^{d}\right)^{T_{N}}$ such that $\tilde{\eta}=\widetilde{\nabla} \phi$ for some $\phi \in \mathbb{R}^{T_{N}}$, $\widetilde{\nabla}$ being as usual the discrete gradient on the torus. Consider the probability measure on $\Gamma_{N}$ defined by

$$
\tilde{v}_{N, v}^{\xi}(\mathrm{d} \tilde{\eta})=\frac{1}{Z_{N, v}^{\xi}} \exp \left(-\frac{1}{2} \sum_{x \in T_{N}} \sum_{i}\left(\pi_{N} \xi\right)_{i}(x)\left(\tilde{\eta}_{i}(x)+v_{i}\right)^{2}\right) m_{N}(\mathrm{~d} \tilde{\eta})
$$

where $m_{N}(\mathrm{~d} \tilde{\eta})$ stands for the image of Lebesgue measure under the map $\phi \rightarrow \widetilde{\nabla} \phi$. Notice that by symmetry $\mathbb{E}_{\tilde{v}_{N, 0}^{\xi}}(\tilde{\eta}(x))=0, x \in T_{N}$. Thus when $v=0, \tilde{v}_{N, 0}^{\xi}$ is the centered Gaussian measure on $\Gamma_{N}$ with covariance $C_{N, i j}^{\xi}$, which in turn, by a well known representation formula (see e.g. [4], Proposition 2.2 for a general statement) is given by

$$
C_{N, i j}^{\xi}(x, y)=\widetilde{\nabla}_{i}\left[\left(-\mathcal{L}_{N}\right)^{-1} \widetilde{\nabla}_{j}^{*} 1_{\{y\}}\right](x),
$$

where $\mathcal{L}_{N}$ has been introduced in (3.3) and $1_{\{y\}}(x)$ is 1 if $x=y$ and is zero otherwise. Equivalently the covariance $C_{N, i j}^{\xi}$ is obtained from gradients of the Green function of the periodized random walk on $T_{N}$ with killing upon hitting the origin (since we are taking gradients the introduction of killing at the origin does not affect our formulas). Now, the linear tilt $v$ only changes the mean in (4.16) and it remains to compute $\mathbb{E}_{\tilde{v}_{N, v}^{\xi}}(\tilde{\eta}(x))$. We write

$$
\mathbb{E}_{\tilde{v}_{N, v}^{\xi}}(\tilde{\eta}(0))=\int_{0}^{1} \frac{\mathrm{d}}{\mathrm{d} t} \mathbb{E}_{\tilde{v}_{N, t v}^{\xi}}(\tilde{\eta}(0)) \mathrm{d} t
$$

But

$$
\begin{aligned}
\frac{\mathrm{d}}{\mathrm{d} t} \mathbb{E}_{\tilde{v}_{N, t v}^{\xi}}\left(\tilde{\eta}_{i}(0)\right) & =-\operatorname{cov}_{\tilde{v}_{N, t v}^{\xi}}\left(\tilde{\eta}_{i}(0), \sum_{x \in T_{N}} \sum_{j}\left(\pi_{N} \xi\right)_{j}(x)\left(\tilde{\eta}_{j}(x)+t v_{j}\right) v_{j}\right) \\
& =-\sum_{x \in T_{N}} \sum_{j}\left(\pi_{N} \xi\right)_{j}(x) v_{j} C_{N, i j}^{\xi}(0, x)=\chi_{N, v}\left(e_{i}\right)-\chi_{N, v}(0) \\
& =\psi_{N, v}^{i}(0, \xi)
\end{aligned}
$$


where in the last line we used the identities (3.5) and (3.7).

Now for every $v \in \mathbb{R}^{d}$ let $v_{N, v}^{\xi}$ be the probability measure induced by $\tilde{v}_{N, v}^{\xi}$ on the field $\eta \triangleq \tilde{\eta}+v$. The above computation shows that $v_{N, v}^{\xi}$ is the Gaussian measure with covariance $C_{N, i j}^{\xi}$ and mean vector

$$
\mathbb{E}_{v_{N, v}^{\xi}}(\eta(x))=v+\psi_{N, v}(x, \xi)
$$

From the convergence of $C_{N, i j}^{\xi}$ we then infer, by Proposition 3.1, that at least along a subsequence $N^{\prime} \rightarrow \infty, v_{N^{\prime}, v}^{\xi} \mu$-almost surely converges weakly to the $v$-tilted state $v_{v}^{\xi}$.

The last observation is a simple identity connecting the diffusion coefficient to surface tension. This type of relation was discussed in $[5,4,7]$ in the context of translation invariant massless fields. Recalling (4.16) we define the surface tension on $T_{N}$ under tilt $v \in \mathbb{R}^{d}$ as

$$
\sigma_{N}^{\xi}(v)=\frac{1}{\left|T_{N}\right|} \log \frac{Z_{N, 0}^{\xi}}{Z_{N, v}^{\xi}} .
$$

By performing the Gaussian integrals and using identities (3.5) and (3.2) one obtains the sought relation

$$
\sigma_{N}^{\xi}(v)=\frac{1}{4}\left(D_{N}(\xi) v, v\right) .
$$

An immediate corollary of Theorem 1.1 is then the a.s. convergence of $\sigma_{N}^{\xi}(v)$ to $\frac{1}{4}(D v, v)$. As we have already mentioned, the corresponding problem raised in [7] in the context of an-harmonic models remains open.

\section{Appendix A}

The proof of Theorem 4.1 is a straightforward adjustment to the discrete Poisson equation (4.3) on $T_{N}$ of the arguments employed in Chapter 9 of [8]: Given $N \in \mathbb{N}$ and a vector field $\vec{g}$ on $T_{N}$ define the distribution function $\eta_{N, \vec{g}}$ of $\vec{g}$ as:

$$
\eta_{N, \vec{g}}(t)=\frac{1}{\left|T_{N}\right|} \#\left\{x \in T_{N}:|\vec{g}(x)|>t\right\} .
$$

By the Marcinkiewicz interpolation theorem applied for the map $T: \vec{f} \mapsto \widetilde{\nabla} u$, where $u$ is the solution of (4.3), it would be enough to check that there exist constants $c_{1}$ and $c_{2}$ such that for every $t \geqslant 0$

$$
\eta_{N, \widetilde{\nabla} u}(t) \leqslant c_{1} \frac{\|\vec{f}\|_{N, 1}}{t}
$$

and,

$$
\eta_{N, \widetilde{\nabla} u}(t) \leqslant c_{2} \frac{\|\vec{f}\|_{N, 2}^{2}}{t^{2}}
$$


Since $\|\widetilde{\nabla} u\|_{N, 2} \leqslant\|\vec{f}\|_{N, 2}$, the second estimate trivially follows from Markov inequality (with $c_{2}=1$ ). It remains to check (A.1)

To facilitate the exposition we shall consider only the dyadic case $N=2^{n}$. Also, it is enough to derive the bound only for the vector fields $\vec{f}$ of the form $\vec{f}(x)=f(x) \vec{e}_{1}$.

For every $k=0,1, \ldots, n-1$ define the decomposition

$$
T_{N}=\bigvee_{x \in K T_{N / K}} B_{x}^{k}
$$

of the torus $T_{N}$ into the lattice boxes $B_{x}^{k}=x+\left\{0, \ldots, 2^{k}-1\right\}^{d}$ of the linear size $K=2^{k}$. The boxes on different $k$-scales are naturally ordered by the inclusion: we say $B_{y}^{l}$ is a predecessor of $B_{x}^{k}$ if $l>k$ and $B_{x}^{k} \subset B_{y}^{l}$. By definition $T_{N}$ is a predecessor of any of the boxes $B_{x}^{k}$ on any of the $k$-scales. Let us fix a number $t>\|f\|_{N, 1}$. We say that a box $B_{x}^{k}$ is correct if

$$
\frac{1}{\left|B_{x}^{k}\right|} \sum_{y \in B_{x}^{k}}|f(y)|=\frac{1}{K^{d}} \sum_{y \in B_{x}^{k}}|f(y)| \leqslant t .
$$

By the choice of $t$ the common ancestor $T_{N}$ is always correct. An incorrect box $B_{x}^{k}$ is called marked if it is incorrect, but all his predecessors are correct. By construction,

$$
t<\frac{1}{\left|B_{x}^{k}\right|} \sum_{y \in B_{x}^{k}}|f(y)| \leqslant 2^{d} t,
$$

for every marked box $B_{x}^{l}$. Since all the marked boxes are disjoint, the first of the inequalities in (A.3) implies that the total marked volume

$$
\frac{1}{\left|T_{N}\right|} \sum_{(k, x): B_{x}^{k} \text { is marked }}\left|B_{x}^{k}\right| \leqslant \frac{\|f\|_{N, 1}}{t} .
$$

Let us renumber all the marked boxes as $B_{1}, \ldots, B_{l}$ and decompose $f$ as

$$
f(x)=f(x) \mathbb{1}_{T_{N} \backslash \cup B_{i}}(x)+\sum_{i=1}^{l} f(x) \mathbb{1}_{B_{i}}(x) \triangleq f^{0}(x)+\sum_{i=1}^{l} f^{i}(x) .
$$

Accordingly, we decompose solutions $u$ of (4.3) as $u=u^{0}+\sum_{1}^{l} u^{j}$, where

$$
\sum_{i=1}^{d} \widetilde{\nabla}_{i}^{*} \widetilde{\nabla}_{i} u^{j}(x)=-\widetilde{\nabla}_{1}^{*} f^{j}(x),
$$

for $j=0,1, \ldots, l$. Evidently,

$$
\eta_{N, \widetilde{\nabla} u}(2 t) \leqslant \eta_{N, \widetilde{\nabla} u^{0}}(t)+\eta_{N, \widetilde{\nabla} \sum_{1}^{l} u^{i}}(t)
$$


Since $\left|f^{0}\right|$ is bounded above by $t$, it follows from (A.2) that

$$
\eta_{N, \widetilde{\nabla} u^{0}}(t) \leqslant \frac{\left\|f^{0}\right\|_{N, 2}^{2}}{t^{2}} \leqslant \frac{\left\|f^{0}\right\|_{N, 1}}{t} .
$$

The bulk of the work is, thus, to derive the $L^{1}$ estimate on the distribution function $\eta_{N, \widetilde{\nabla} \sum_{1}^{l} u^{i}}$ corresponding to the irregular part $\sum_{1}^{l} f^{l}$ of the vector field $\vec{f}=f \vec{e}_{1}$. Eq. (A.5) feels the right-hand side $f^{i}$ only inside the box $B_{i}$. For $B_{i}=B_{x}^{k}$ let us define the enlargement $\bar{B}_{i}=\bar{B}_{x}^{k}$ via

$$
\bar{B}_{i}=\bar{B}_{x}^{k}=\bigcup_{y \in K T_{N / K}:\|y-x\|_{\infty} \leqslant K} B_{y}^{k} .
$$

In other words, $\bar{B}_{x}^{k}$ is the union of $B_{x}^{k}$ with all its nearest and next-nearest neighbour boxes on the $k$ th scale. By (A.4),

$$
\frac{1}{\left|T_{N}\right|} \sum_{i=1}^{l}\left|\bar{B}_{i}\right| \leqslant 3^{d} \frac{\|f\|_{1}}{t} .
$$

In order to estimate $\eta_{N, \widetilde{\nabla} u^{i}}$ outside $\bar{B}_{i}$ write:

$$
\widetilde{\nabla}_{j} u^{i}(x)=\sum_{y \in B_{i}} \widetilde{\nabla}_{1} \tilde{\nabla}_{j} G_{N}(x-y) f^{i}(y) \triangleq \sum_{y \in B_{i}} \widetilde{\nabla}_{1, j}^{2} G_{N}(x-y) f^{i}(y)
$$

where

$$
\widetilde{\nabla}_{1, j}^{2} G_{N}(z)=\sum_{m=0}^{\infty} \widetilde{\nabla}_{1, j}^{2} p_{m}(z),
$$

and $p_{m}$ is the $m$-step transition function of the simple random walk on $T_{N}$.

There is no loss to assume that $f^{i}$ has zero average:

$$
\sum_{y \in B_{i}} f^{i}(x)=0
$$

Thus, for $B_{i}=B_{y_{0}}^{k}$, we can rewrite (A.8) as

$$
\widetilde{\nabla}_{j} u^{i}(x)=\sum_{y \in B_{i}}\left(\widetilde{\nabla}_{1, j}^{2} G_{N}(x-y)-\widetilde{\nabla}_{1, j}^{2} G_{N}\left(x-y_{0}\right)\right) f^{i}(y) .
$$

By Theorem 1.5.5 in [13] $(d \geqslant 3)$ or by Theorem 1.6.5 in [13] $(d=2)$,

$$
\left|\widetilde{\nabla}_{1, j}^{2} G_{N}(x-y)-\widetilde{\nabla}_{1, j}^{2} G_{N}\left(x-y_{0}\right)\right| \leqslant c_{3}(d) \frac{K}{\left|x-y_{0}\right|^{d+1}} .
$$

It follows that

$$
\sum_{x \in T_{N} \backslash \bar{B}_{i}}\left|\widetilde{\nabla} u^{i}(x)\right| \leqslant c_{4} \sum_{y \in B_{i}}|f(y)|
$$


for all $i=1, \ldots, l$. Since the marked boxes $B_{1}, \ldots, B_{l}$ are disjoint,

$$
\sum_{x \in T_{N} \backslash \cup \overline{B_{i}}}\left|\widetilde{\nabla} \sum_{i=1}^{l} u^{i}(x)\right| \leqslant \sum_{y \in T_{N}}|f(y)| .
$$

As a result,

$$
\frac{1}{\left|T_{N}\right|} \#\left\{x \in T_{N} \backslash \bigcup \bar{B}_{i}:\left|\widetilde{\nabla} \sum_{i=1}^{l} u^{i}(x)\right|>t\right\} \leqslant c_{4} \frac{\|f\|_{N, 1}}{t},
$$

which, by (A.7), leads to the desired estimated on the distribution function of the $\widetilde{\nabla} \sum_{1}^{l} u^{i}$ part:

$$
\eta_{N, \widetilde{\nabla} \sum_{1}^{l} u^{i}}(t) \leqslant c_{5} \frac{\|f\|_{N, 1}}{t}
$$

The proof of Theorem 4.1 is concluded.

\section{REFERENCES}

[1] D. Boivin, Weak convergence for reversible random walks in a random environment, Ann. Probab. 21 (1993) 1427-1440.

[2] D. Bovin, Y. Derriennic, The ergodic theorem for additive cocycles of $\mathbb{Z}^{d}$ or $\mathbb{R}^{d}$, Ergodic Theory Dynam. Systems 11 (1991) 19-39.

[3] A. De Masi, P. Ferrari, S. Goldstein, W.D. Wick, An invariance principle for reversible Markov processes. Applications to random motions in random environments, J. Statist. Phys. 55 (1989) 787-855.

[4] J.-D. Deuschel, G. Giacomin, D. Ioffe, Large deviations and concentration properties for $\nabla \phi$ interface models, Probab. Theory Related Fields 117 (2000) 49-111.

[5] T. Funaki, H. Spohn, Motion by mean curvature from the Ginzburg-Landau $\nabla \phi$ interface model, Comm. Math. Phys. 185 (1997) 1-36.

[6] H.O. Georgii, Gibbs Measures and Phase Transitions, in: de Gruyter Studies in Math., Vol. 9, 1988.

[7] G. Giacomin, S. Olla, H. Spohn, Equilibrium fluctuation for a Ginzburg-Landau interface model, Ann. Probab., to appear.

[8] D. Gilbarg, N.S. Trudinger, Elliptic Partial Differential Equations of Second Order, Springer, 1983.

[9] V.V. Jikov, S.M. Kozlov, O.A. Oleinik, Homogenization of Differential Operators and Integral Functionals, Springer, 1994.

[10] C. Kipnis, S.R.S. Varadhan, Central limit theorem for additive functionals of reversible Markov processes and applications to simple exclusions, Comm. Math. Phys. 104 (1986) $1-19$.

[11] S.M. Kozlov, The method of averaging and walks in inhomogeneous environments, Russian Math. Surveys 40 (2) (1985) 73-145.

[12] C. Landim, S. Olla, S.R.S. Varadhan, Finite-dimensional approximation of the self-diffusion coefficient for the exclusion process, Ann. Probab., to appear.

[13] G.F. Lawler, Intersection of Random Walks, Probability and its Applications, Birkhäuser Boston, 1991. 
[14] R. Künnemann, The diffusion limit for reversible jump processes on $Z^{d}$ with ergodic random bond conductivities, Comm. Math. Phys. 90 (1983) 27-68.

[15] N.C. Meyers, An $\mathbb{L}_{p}$ estimate for the gradient of solutions of second order elliptic divergence equations, Ann. Sc. Norm. Sup. Pisa 17 (1963) 189-206.

[16] S. Olla, Lectures on Homogenization of Diffusion Processes in Random Fields, Ecole Polytechnique, 1994.

[17] H. Owhadi, Approximation of the effective conductivity of ergodic media by periodization, Preprint, 2002.

[18] G. Papanicolaou, S.R.S. Varadhan, Diffusions with random coefficients, in: Statistics and Probability: Essays in Honor of C.R. Rao, North-Holland, Amsterdam, 1982, pp. 547-552.

[19] M. Talagrand, A new look at independence, Ann. Probab. 24 (1996) 1-34. 